\title{
Design and Implementation of Advanced Internet Management System
}

\author{
Chia-Sheng Tsai ${ }^{1 *}$, and Cheng-Wei Lin
}

\begin{abstract}
With the rapidly growing on Internet and variety applications through Internet, user reliabilities on Internet raise dramatically. Relatively, due to the convenience, users easily consume the resources without considerations. How to monitor and analyze the network traffic becomes an important lesson. In order to make MIS to manage numbers of servers and network switches efficiently to dig out network traffic abnormality, numbers equipment choices are in the market, but usually the equipment only manages and monitors on appointed application due to limited hardware. The cost is also extremely high. Most of all, equipments functionalities may not satisfy MIS requirements because of limited cost, which makes MIS need to manage and monitor several system at the same time.

This research provides a monitoring and analyzing system to collect network traffic information. In order to make MIS uses easily, this system is running through SNMP protocol and integrating network monitoring instructions. The monitoring equipment is taking advantage from Cisco Catalyt 2950 with Open Source Network Development software, which efficiently collects and analyzes the abnormality on local network traffic,enables MIS to get local network traffic information and abnormal usages on each channel through webpage conveniently. This system is useful MIS to observe and analyze the network traffic.
\end{abstract}

Keywords - Cisco Catalyt 2950、Open Source 、 SNMP 、 Server - Switch

\section{INTRODUCTION}

$\mathrm{W}$ ITH the popularity of Internet services, on the web, more and more diverse, leading to a growing dependence on the Internet, but also makes more and more abuse of network resources, so how effective monitoring network has become now the most popular topics, network monitoring and network managers have the responsibility and relatively increased, network administrators must always monitor the status of network equipment and services of the host, and always monitor network traffic, quickly understand whether there is suffering to malicious attacks and the use of abnormal network segment. Many commercial software and free software available in the market, although monitoring services, but these commercial software only detect restrictions in one area or need hardware, and even some commercial software will be charged separately for each function module, which the cost is a big cost considerations. In addition, these business software for different monitoring equipment and monitoring purposes is somewhat limited. These monitoring systems, such as: ... and so on, only

Chia-Sheng Tsai ${ }^{1}$ is with the Tatung University, Taipei, Taiwan. for certain surveillance monitoring and management, but can not meet the various needs of network administrators, network administrators that sets monitoring system must be installed alarms error rate greatly improved, and general business software is also unable to detect the network traffic into usable data storage even archives, it led to the monitoring system for network managers must monitor every one of them, greatly improve the human cost and time.

The study first discusses general commercial software or freeware, which features are provided, and subject to what restrictions. In addition, we propose a set of network segments to monitor Cisco Switch Monitoring System flow data, and provides a tight network abnormal traffic report and daily reporting capabilities and real-time output of network traffic charts, so network managers this system can be used to easily monitor LAN traffic, greatly reducing cost and time-consuming labor.

\section{MRTG}

\section{A. MRTG Scheme}

MRTG is a wide scope of application traffic statistics tool, MRTG needs by way of SNMP or plug-ins to collect data, generate real-time charts, usually set to 5 minutes once. MRTG is based on SNMP protocol to ask Router, Switch, Firewall...etc, and then pass back values displayed as charts. We can also execute a plug-in, the program will pass back to the statistical value, then MRTG is responsible for displaying a chart. MRTG biggest limitation is that your Router, Switch, Firewall ... etc. Otherwise it is impossible to get the actual query traffic. MRTG is not a solution in the form NMS, MRTG is at best just a Polling Engine only, it is a good draw diagrams, as NMS general, MRTG will be get the operation, but it is not designed to detect and solve problems.

The SNMP protocol MRTG is to ask Router, Switch, Firewall ... etc, and then pass back values displayed as charts. We can also execute a plug-in, the program will pass back to the statistical value, then MRTG is responsible for displaying a chart. The following diagram (Figure 2-1) the result of the test MRTG network packets.

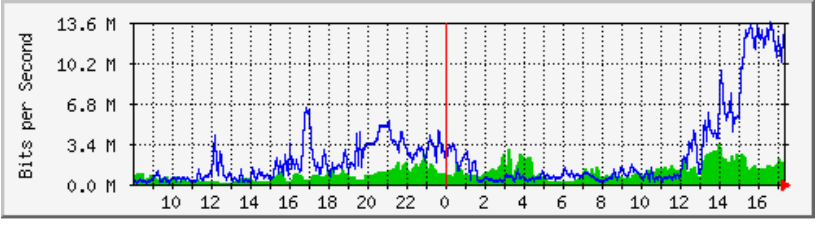

Fig. 2-1 MRTG graph 


\section{B. SNMP Introduction}

SNMP provides a simple operating procedures, so that managers can use to manage the use of TCP / IP protocol on the network device, it can supervise management devices from different manufacturers, for any operating system [10] [11]. SNMP forms part of the Internet Agreement Internet Engineering Task Force (IETF, Internet Engineering Task Force) definition. The agreement can support the network management system to monitor the connection to the device on the network whether there is any cause upper management concern. It consists of a set of network management standard composition, comprising an application layer protocol (Application Layer Protocol), the database model (Database Schema), and a set of data objects [3].

An SNMP managed network consists of three key elements:

- NMSs (Network-management systems)

- Managed Device

- Agent

SNMP (Simple Network Management Protocol) is the mainstream variety of network management protocol does not require complicated installation process and expertise, you can do network management. SNMP network management architecture consists mainly SNMP, SMI and MIB and other three parts together.

-SET: management server to change the settings in the management of information by the device.

-GET: Management Server Release capture by setting management information within the device.

-TRAP: a managed device when a specific error occurs, will take the initiative to issue a warning message to the management server.

\section{SoftPerfect Networx Portable Introduction}

SoftPerfect NetWorx Portable is a free and powerful Chinese network traffic monitoring software, the main function of the software can be divided into two, one is monitoring network traffic statistics, one is the Internet upload and download bandwidth limit function! We can understand our current network upload and download status instantly through it. But also provides a daily report, weekly reports and monthly reports, allowing us to examine network traffic conditions. There is also provided a Trace and Netstat function.

\section{Network traffic Monitor Introduction}

Network Traffic Monitor is a packet analyzer that lets you capture, view and analyze network traffic. This tool is very easy to query network problems and applications. Key features include support for more than 300 public and Microsoft agreement, while capturing conversation, wireless monitoring mode and the other being promiscuous mode traffic.

\section{E. Network traffic Monitor Introduction}

Iperf is a TCP / IP and UDP / IP performance measurement tool that provides information on network throughput, as well as shock, packet loss rate, the maximum segment size and maximum transmission unit and other statistical information; which can help us to test network performance, locate network bottlenecks. When you need to test whether the speed of the Internet environment, the ideal situation, the most common is the interest My Network Places, FTP transmission to achieve the purpose of testing, but if you need to repeat the test to obtain mean, this measurement method may be relatively ineffective, so there is such a tool iperf test for network transmission speed is just more convenient. To use iperf must have two computers, one for the server side, and the other as the client to the environment as a demonstration to the windows, you need to download windows version can be made here, then other platforms, iperf have to provide source codeIt can be used for other platforms can be used to recompile. The following is Iperf test, Figure 2-2 Iperf Server (hereinafter referred to as servo end), Figure 2-3 for the Iperf Client (hereinafter referred to as the client), servo-side execution "iperf -s" listening client the implementation of "iperf -c 10.1.1.1 -w 50M -t 120 -i 10", - c is Server-side IP, -w file size for testing, -t to monitor the measurement data is 120 seconds, -i to every 10 seconds will display the data through a simple set-up and simple instructions we easily transfer speed measurement for bandwidth between two nodes within the network, of course iperf program also supports a number of parameters, we can refer to its specific setting HELP, comparison of commonly used buffer size -1, -m mtu display port tcp maximum value, - $p$ specifies the port used by the servo end or client is connected, -u uses the udp protocol, -w specifies the TCP window size, the default is $8 \mathrm{~KB}$. Also on the client can specify the number of bytes transferred $-n$, -r separate two-way transmission testing, you can run the servo side iperf by $-D$ parameters as a service to $-\mathrm{R}$ to stop iperf service.

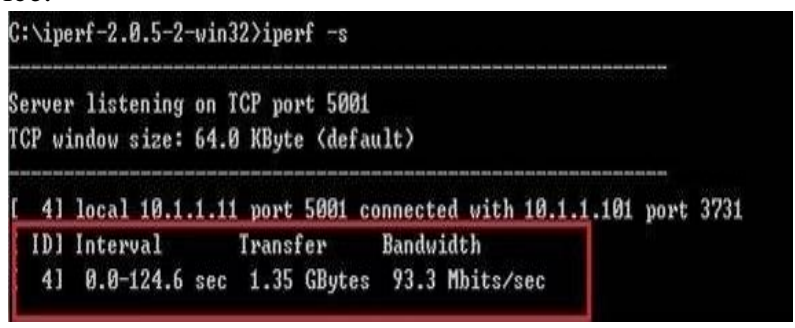

Fig. 2-2 Iperf Server

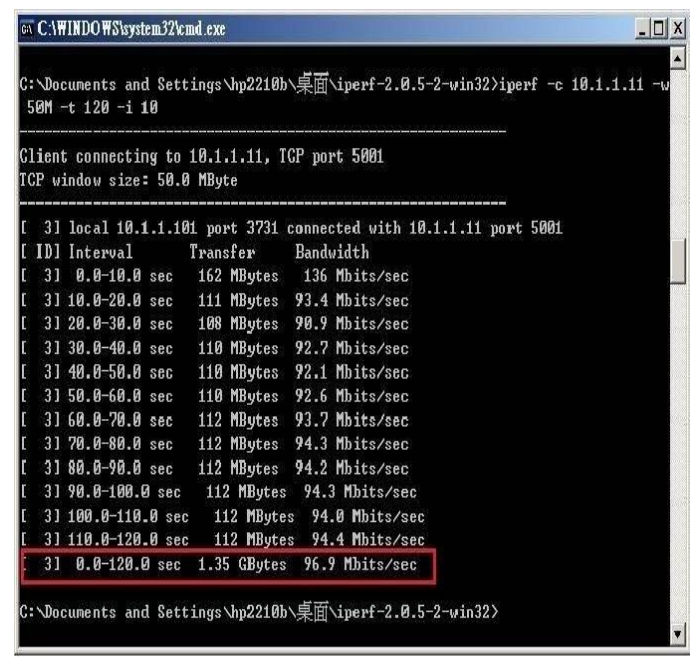

Fig. 2-3 Iperf Client 


\section{F. Cisco Monitor Introduction}

Cisco Switch any problems will affect users, monitor the implementation of preventive measures can help you identify problems early and avoid potential problems.

With monitoring each of the segments on / off, you can get the state of switch ports and usage. You can proactively monitor the switch and its ports and quickly notify users switch port fault or switch. Monitoring the status and performance of the switch can switch through the device manager to evaluate. As can be seen in Figure 2-4 below number of packets and packet size Interfaces fastEthernet sent by 0/4, it can be seen in Figure 2-5 Interfaces fastEthernet $0 / 2$ Number of received packets and packet size, which is also aware of its switch state.

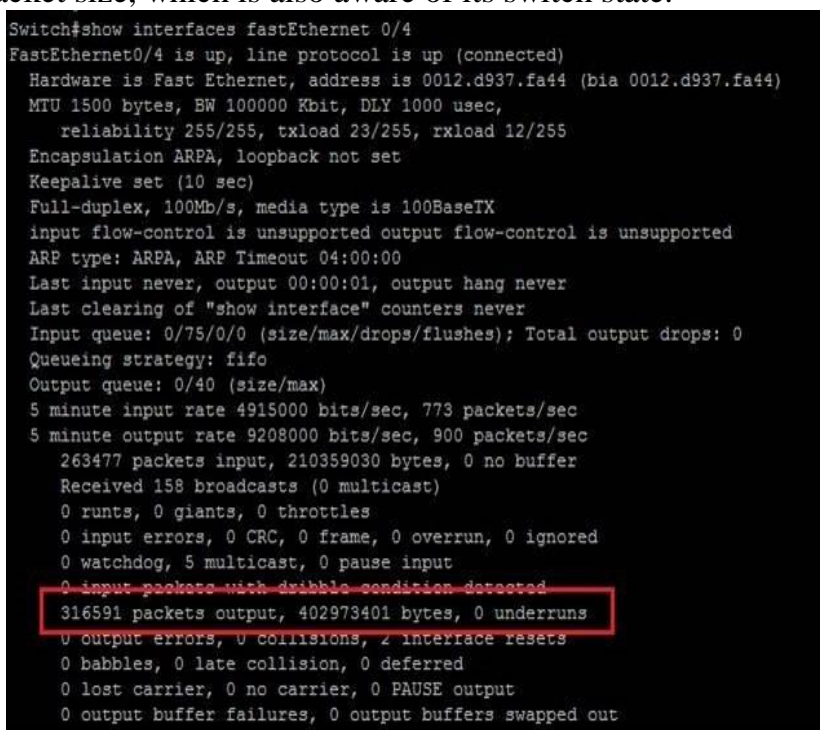

Fig. 2-4 Internet fastEthernet0/4 Status

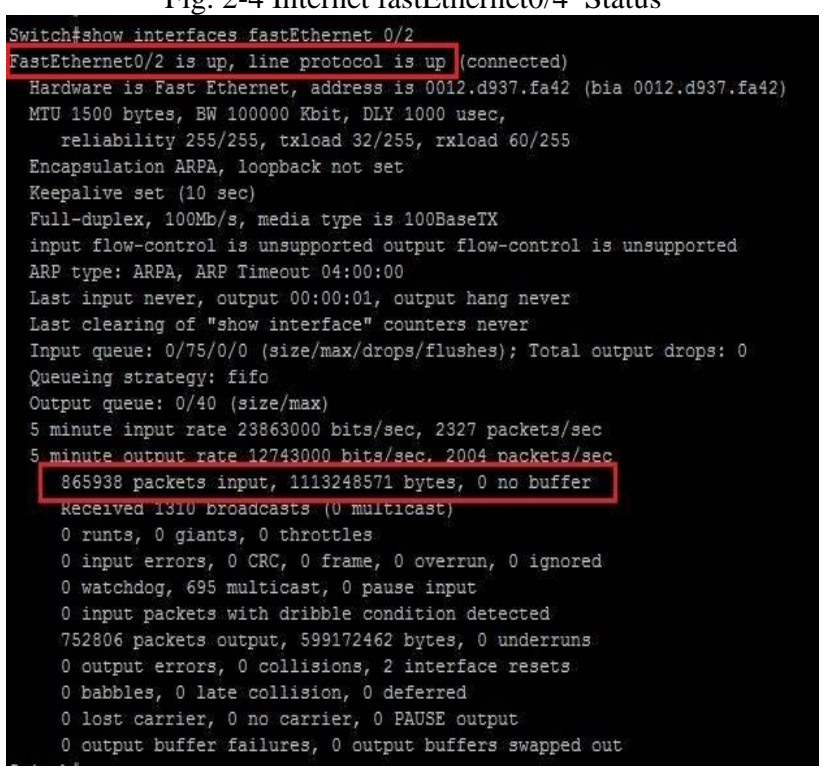

Fig. 2-5 Internet fastEthernet0/2 Status

\section{PROPOSED SCHEME}

\section{A. System Model}

Continuously push forward the construction of information has led to the growing popularity of LAN and the Internet. Network management software to monitor and control should have two basic functions. With the rapid advances in network technology, the transmission bandwidth continue to raise up, causing improper use of network resources a growing problem, and therefore network monitoring and network management more and more attention. Accurate and complete network monitoring data, can help network administrators understand resource usage and user internet usage behavior for network resource planning and information security processing. The traffic monitoring is now an integral MIS, MIS can clearly inform the user if the upper limit is exceeded, and can quickly alert network Yong plug, to distinguish legitimate or malicious network traffic so that it caused paralysis, how to effectively manage the web various network segments and devices, and understanding of the issues timely warning of symptoms, improve service quality and efficiency of the Internet, so that the external network bandwidth control and traffic monitoring and analysis is becoming increasingly important. The system architecture is shown below, network monitoring software using SNMP to collect every minute of each port of Cisco network status and network traffic, and save data to MySQL database accessible and information displayed on the page, let network administrators can more easily view and analyze network usage on the Web interface, but also its data output to the report, in order to facilitate network administrators can view.

\section{B. System Architecture}

To allow network managers can understand short network architecture and management, and reduce the burden of network management personnel, WEB interface is one of the most easy to use and easy to understand the platform, even if not under the command of managers, and still can easily operate. The problem occurs when the equipment, if not in charge of people may not know where the device is placed on, and usually takes a lot of time to confirm the location of the device. But after using WEB interface can quickly understand which network traffic anomaly, the system can automatically shut down the network segment. This will greatly reduce the burden of network management personnel. The following diagram (Figure 3-1) will be described for the four sub-system architecture. In this study, we propose the system integration of network management systems.

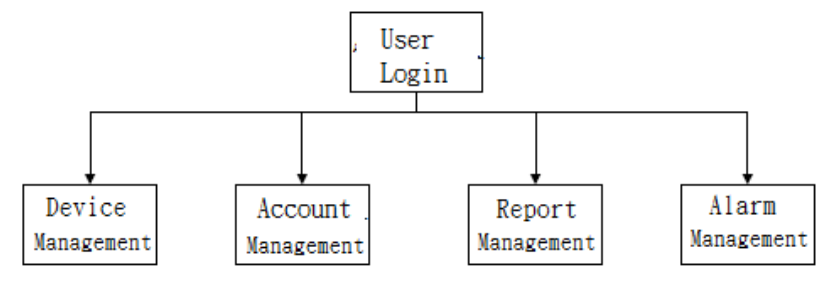

Fig. 3-1 System Architecture

\section{IMPLEMENTATION}

\section{A. Report Management}

If in hours, starting and ending time input For further information must be YYYYMMDDHH (Figure 4-1), the system will enter the hour of data are combined into a total data of each communication port (Figure 4-1\&2), where port for the 
communications port number, PackageNum communication port traffic for the time interval, Traffic Radio in the time interval of the overall flow of traffic to the total percentage.

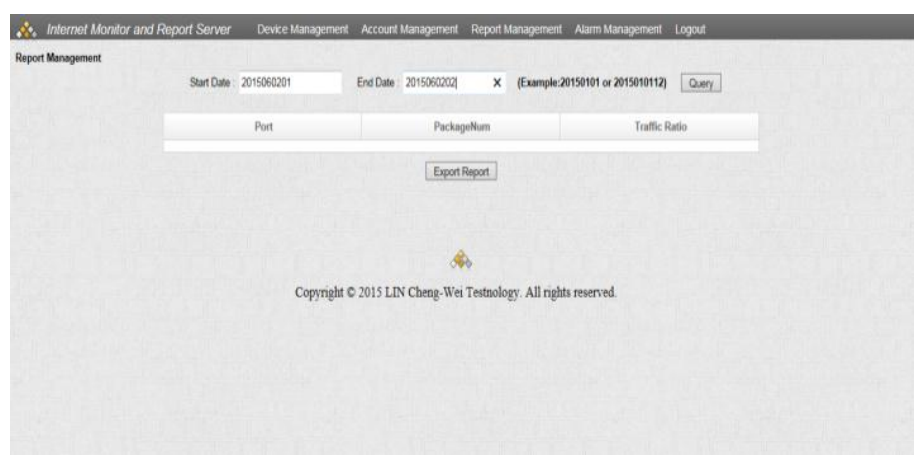

Fig. 4-1: Input the beginning and ending time of hours

Whether on the unit or when units of days, all search results can be output csv file manually, and the system will be in daily two points, automatically output reports in order to facilitate network administrators to backup file every day. The csv file as follows (Figure 4-3).

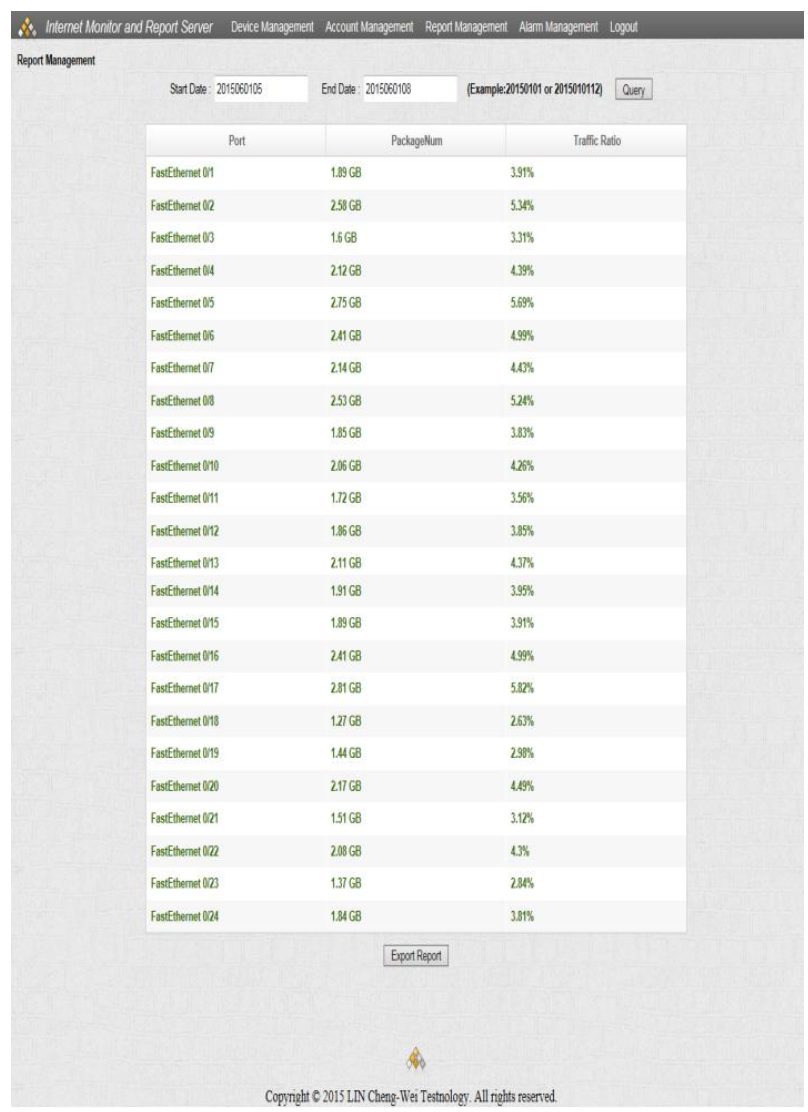

Fig. 4-2: Results to query data of hour

\begin{tabular}{|c|l|l|l|}
\hline \multicolumn{1}{|c|}{ A } & \multicolumn{1}{|c|}{$\mathrm{B}$} & \multicolumn{1}{|c|}{$\mathrm{C}$} \\
\hline 1 & Port Name & Package & Package Percent \\
\hline 2 & FastEthemet 0/1 & $1.89 \mathrm{~GB}$ & $3.91 \%$ \\
\hline 3 & FastEthemet 0/2 & $2.58 \mathrm{~GB}$ & $5.34 \%$ \\
\hline 5 & FastEthemet 0/3 & $1.6 \mathrm{~GB}$ & $3.31 \%$ \\
\hline 6 & FastEthemet 0/4 & $2.12 \mathrm{~GB}$ & $4.39 \%$ \\
\hline 7 & FastEthemet 0/5 & $2.75 \mathrm{~GB}$ & $5.69 \%$ \\
\hline 8 & FastEthemet 0/6 & $2.41 \mathrm{~GB}$ & $4.99 \%$ \\
\hline 9 & FastEthemet 0/7 & $2.14 \mathrm{~GB}$ & $4.43 \%$ \\
\hline 10 & FastEthemet 0/8 & $2.53 \mathrm{~GB}$ & $5.24 \%$ \\
\hline 11 & FastEthemet 0/9 & $1.85 \mathrm{~GB}$ & $3.83 \%$ \\
\hline 12 & FastEthemet 0/10 & $2.06 \mathrm{~GB}$ & $4.26 \%$ \\
\hline 13 & FastEthemet 0/11 & $1.72 \mathrm{~GB}$ & $3.56 \%$ \\
\hline 14 & FastEthemet 0/13 & $1.86 \mathrm{~GB}$ & $3.85 \%$ \\
\hline 15 & FastEthemet 0/14 & $1.91 \mathrm{~GB}$ & $4.37 \%$ \\
\hline 16 & FastEthemet 0/15 & $1.89 \mathrm{~GB}$ & $3.95 \%$ \\
\hline 17 & FastEthemet 0/16 & $2.41 \mathrm{~GB}$ & $3.91 \%$ \\
\hline 18 & FastEthemet 0/17 & $2.81 \mathrm{~GB}$ & $4.99 \%$ \\
\hline 19 & FastEthemet 0/18 & $1.27 \mathrm{~GB}$ & $5.82 \%$ \\
\hline 20 & FastEthemet 0/19 & $1.44 \mathrm{~GB}$ & $2.63 \%$ \\
\hline 21 & FastEthemet 0/20 & $2.17 \mathrm{~GB}$ & $2.98 \%$ \\
\hline 22 & FastEthemet 0/21 & $1.51 \mathrm{~GB}$ & $4.49 \%$ \\
\hline 23 & FastEthemet 0/22 & $2.08 \mathrm{~GB}$ & $3.12 \%$ \\
\hline 24 & FastEthemet 0/23 & $1.37 \mathrm{~GB}$ & $4.30 \%$ \\
\hline 25 & FastEthemet 0/24 & $1.84 \mathrm{~GB}$ & $2.84 \%$ \\
\hline & & $3.81 \%$ \\
\hline
\end{tabular}

Fig. 4-3 CSV file

\section{B. Alarm Management}

If the device has traffic anomaly, exception management office listed in the communications port traffic anomaly, with this list know which communication port in question. To allow managers to quickly understand traffic anomaly point in time, so that service disruption time compressed into the shortest possible time, the event will occur is stored in the database in the future as to assess the availability of analytical information, this information may also be used as an important reference data to determine whether the equipment or system needs to be replaced.

For network managers can define your own alarm traffic volume, refer to the daily report data settings, alarm data will be written to the database accessible to facilitate network administrators query, the system will send alarm information to the specified mail to inform managers, is also set to send the designated mail, alarm management in the following figure (Figure 4-16). 


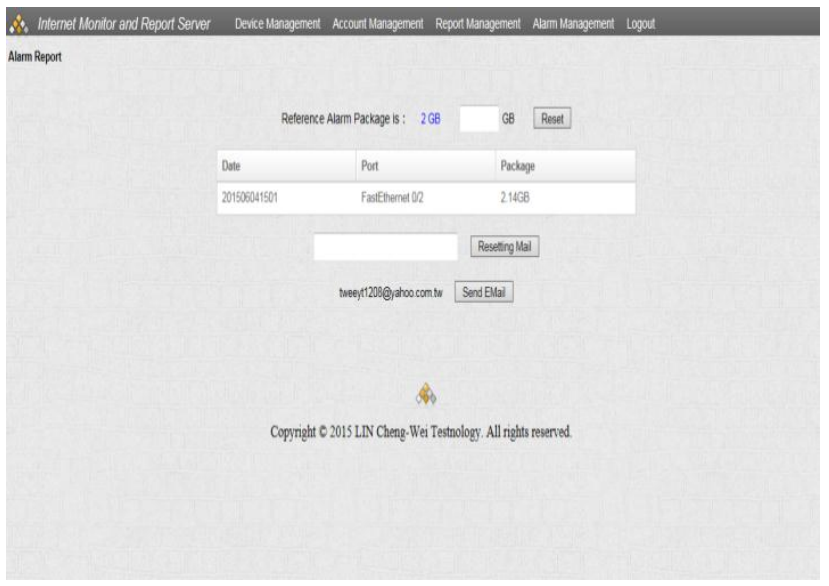

Fig. 4-4 Alarm Management

\section{CONCLUSIONS}

In this study, data collection, their equipment is monitored and managed. With services available on the Internet, more and more managers responsible for servers and network equipment increasing, in general, managers if you want to control all network equipment and servers, usually detected through a variety of ways and different systems in order to confirm the situation, a single type of network monitoring and management software has been unable to meet the needs of management personnel. Although there are two switches integrated server and as one of the monitoring and management system, but need to consider the installation of hardware limitations and maintenance costs and other factors.

The management personnel responsible for the maintenance and operation of network traffic equipment are unclear, often because of network traffic anomalies and spend too much time looking at anomalies and follow-up treatment. To allow managers to have a platform to grasp the situation clear and concise manner the operation of online systems and equipment, as well as enhance the protection of information security, so I decided to construct network traffic collection and monitoring and analysis system, hoping to assist this system can guarantee maintenance and operation of the equipment and systems are not threatened by the various aspects of availability, making it possible to minimize the damage. When an event occurs the system immediately send a message to management, and are also provided daily reports and real-time network traffic charts, assist managers problem determination and maintenance and shorten the duration of abnormal flow.

\section{REFERENCES}

[1] WAMP,http://sofree.cc/windows-appserv/

[2] Apache Tomcat,http://tomcat.apache.org/

[3] SNMP http://en.wikipedia.org/wiki/Simple_Network_Management_Protocol

[4] MySQL,http://www.mysql.com/

[5] Spring, http://projects.spring.io/spring-framework/ 